\title{
Range-Separated Hybrid Density Functional Study of Organic Dye Sensitizers on Anatase $\mathrm{TiO}_{2}$ Nanowires
}

\author{
Hatice Ünal, ${ }^{\dagger}$ Deniz Gunceler, ${ }^{\ddagger}$ Oğuz Gülseren, ${ }^{\text {II }}$ Şinasi Ellialtığlu, ${ }^{\S}$ and Ersen Mete ${ }^{*}, \dagger$ \\ ${ }^{\dagger}$ Department of Physics, Balkesir University, Balkesir 10145, Turkey \\ ${ }^{\ddagger}$ Department of Physics, Cornell University, Ithaca, New York 14853, United States

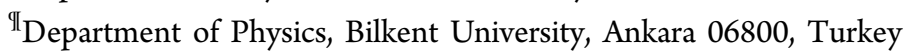 \\ ${ }^{\S}$ Basic Sciences, TED University, Ankara 06420, Turkey
}

ABSTRACT: The adsorption of organic molecules coumarin and the donor- $\pi$ acceptor type tetrahydroquinoline (C2-1) on anatase (101) and (001) nanowires has been investigated using screened Coulomb hybrid density functional theory calculations. Simple coumarin core forms a single bond with the $\mathrm{TiO}_{2}$. C2-1 exhibits a bidentate mode giving rise to much stronger adsorption energies on the nanowire surface. Nonlinear solvation effects on the binding characteristics of the dye chromophores on the nanowire facets have also been examined. These two dye sensitizers show different electronic charge distributions for the highest occupied and the lowest unoccupied molecular states. We studied the electronic structures in terms of the positions of the band edges and adsorbate-related gap states and their effect on the absorption spectra of the dye-nanowire combined systems. These findings were interpreted and discussed from the viewpoint of better light harvesting and charge separation as well as in relation to more

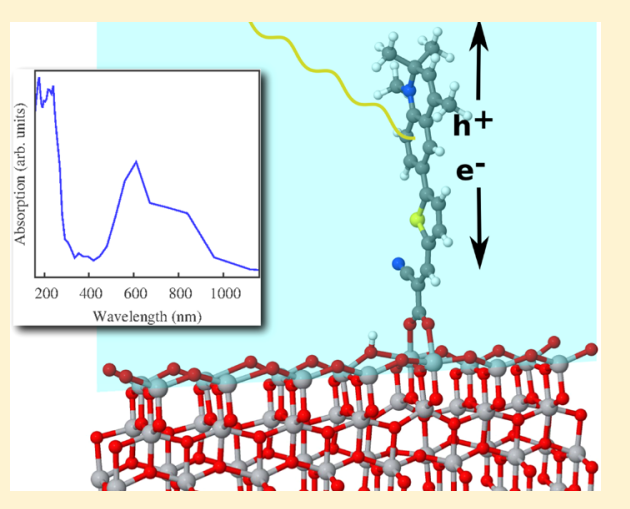
efficient charge carrier injection into the semiconductor nanowire.

\section{INTRODUCTION}

Recently, dye-sensitized solar cells (DSSCs) have become an important research field in direct energy generation from sunlight. In a typical system, dye adsorbates on $\mathrm{TiO}_{2}$ nanostructures are responsible for light harvesting with their highest occupied molecular orbitals (HOMOs) residing in the band gap of the host semiconductor. The coupling of their lowest unoccupied molecular orbital (LUMO) with the conduction band (CB) of $\mathrm{TiO}_{2}$ serves as a natural pathway for photogenerated charge injection from the dye to the $\mathrm{CB}$ of the substrate. The cell is then regenerated by interaction of the excited dye with a redox couple. ${ }^{1,2}$ The open-circuit voltage of the cell, $V_{\mathrm{OC}}$, is the difference between the highest occupied level of the dye-semiconductor system and redox potential of the mediator (typically iodide). For such a solar cell construction, titania has one of the most appropriate band positions among the other wide-bandgap semiconductors.

$\mathrm{TiO}_{2}$, particularly the (001) surface of the anatase phase, shows excellent photocatalytic activity under ultraviolet (UV) irradiation. ${ }^{2,3}$ Sensitizer chromophores not only drive the UVlimited photoresponse of $\mathrm{TiO}_{2}$ into the visible range but also play an important role in charge carrier dynamics. In fact, the overall cell efficiency depends on the preferable properties of the dye-semiconductor composite system in relation to the factors such as the photoelectric conversion, charge carrier injection, electron-hole recombination rates, and charge transport performance.

High surface-to-volume ratio of the semiconductor material is another component of the efficiency consideration. In general, $\mathrm{TiO}_{2}$ nanoparticles provide multiple surfaces exposing large number of active sites. On the other hand, quasi-onedimensional titania nanowires ${ }^{4}$ not only accommodate even larger areas but also are superior in n-type conductivity, reducing the photogenerated charge recombination rates. In the forms of nanostructures, thermodynamically the most stable phase of $\mathrm{TiO}_{2}$ is the anatase polymorph. ${ }^{5-7}$ The interaction between anatase nanostructures and dye sensitizers is one of the basic issues to improve the efficiency of DSSCs.

The choice of the sensitizer dye depends not only on its durability, absorption, and charge injection ability during the energy conversion cycles, but also on the cost and ease of its production. Ru-based photosensitizers, reaching up to $11 \%$ efficiencies (under AM 1.5 illumination), constitute an important part of the dye complexes that are used in DSSC technology. ${ }^{1,8-15}$ Researchers have focused on finding alternatives to those well-known, metal-driven, and relatively more expensive dye sensitizers. ${ }^{16}$ Several organic dye molecules have been shown to be strikingly efficient in light harvesting. ${ }^{17-25}$ For instance, indoline derivatives showed $9.52 \%$ efficiency. ${ }^{26}$ High efficiencies of the $\mathrm{Ru}$ complexes are attributed to their high charge injection rates into the conduction band (CB) of the $\mathrm{TiO}_{2}$ pertaining to their metal-to-ligand charge-transfer ability. Novel organic donor- $\pi$-acceptor dyes like tetrahydro-

Received: August 5, 2014

Revised: September 30, 2014

Published: September 30, 2014 
quinoline-based $\mathrm{C} 2-1^{24,25}$ have been proposed for achieving intramolecular charge separation.

Improvements in the dye design crucially depend on the deep understanding of the fundamental properties of the sensitizer and its interaction with the nanostructured semiconductor. Quantitatively, novel organic dye molecules with advanced chemical and physical properties can be tailored with the aid of theoretical modeling. ${ }^{27-29}$ Theoretical studies have focused on the prediction of the electronic structure of the chromophores and of their interface with the $\mathrm{TiO}_{2}$ substrates. $^{30-40}$ In particular, the family of tetrahydroquinoline derived dyes have been studied with ab initio calculations. ${ }^{41}$ O'Rourke et al. have also considered these dyes on the $\mathrm{TiO}_{2}(101)$ surface to predict their electronic structures. ${ }^{42}$

In this study, we aimed to understand the binding modes, electronic structures, and optical spectra of dye sensitizers on anatase $\mathrm{TiO}_{2}$ nanowires having (101) and (001) facets by standard as well as hybrid density functional theory (DFT) calculations. Tetrahydroquinoline-based C2-1 $\left(\mathrm{C}_{21} \mathrm{H}_{20} \mathrm{~N}_{2} \mathrm{SO}_{2}\right)$ organic dyes achieving directional charge distribution upon photoexcitation have been considered as the light-harvesting molecules adsorbed on the nanowires. To better describe the differences from a simple dye molecule, we also included coumarin $\left(\mathrm{C}_{9} \mathrm{H}_{6} \mathrm{O}_{2}\right)$ without the anchoring carboxylic ligands. Conventional coumarin dyes are extensively studied in the literature. ${ }^{19,20,37,43-57}$ Because DSSC operates in solution, the solvation effects become important. Therefore, we addressed this by using a new polarizable continuum model (PCM) for solvents with different ionicities. After briefly describing the computational methods, we will discuss the results in detail.

\section{COMPUTATIONAL DETAILS}

To investigate the geometric and electronic properties of the dye and nanowire composite systems, we performed pseudo potential plane wave calculations based on standard and hybrid DFT using both Perdew-Burke-Ernzerhof $(\mathrm{PBE})^{58}$ and Heyd-Scuseria-Ernzerhof (HSE) $)^{59-61}$ exchange-correlation (XC) functionals as implemented in the Vienna ab initio simulation package (VASP). ${ }^{62}$ The ionic cores and valence electrons with an energy cutoff value of $400 \mathrm{eV}$ for the plane wave expansion were treated by projector-augmented waves (PAW) method. ${ }^{63,64}$ The convergence of our calculated values have been carefully tested with respect to $k$-point sampling.

In addition to the standard DFT, we used the rangeseparated hybrid density functional approach that partially admixes exact Fock exchange and PBE exchange energies. These type of hybrids offer a better description of localized $d$ states and improve the energy gap related features over the standard XC schemes. Range-separated hybrid functionals tend to compensate the localization deficiency due to the lack of proper self-interaction cancellation between the Hartree and exchange terms of the standard DFT. Correct description of the adsorbate-driven gap states around the band edges is critical for the estimation of the photovoltaic properties. In HSE functional, the exchange energy is separated into two parts as the long-range (LR) and the short-range (SR) as

$$
E_{\mathbf{X}}^{\mathrm{HSE}}=a E_{\mathbf{X}}^{\mathrm{HF}, \mathrm{SR}}(\omega)+(1-a) E_{\mathrm{X}}^{\mathrm{PBE}, \mathrm{SR}}(\omega)+E_{\mathrm{X}}^{\mathrm{PBE}, \mathrm{LR}}(\omega)
$$

where $a$ is the mixing coefficient ${ }^{65}$ and $\omega$ is the range separation parameter. ${ }^{59-61}$ In this approach, the correlation part of the energy is taken from standart PBE. ${ }^{58}$
To study the effect of the solvent (chloroform or water) on the electronic structure of the dye-nanowire combined systems, we carried out calculations using the nonlinear polarizable continuum model (PCM) implemented in the open-source code JDFTx. ${ }^{66-69}$ Polarizable continuum models surround the solvated species with a continuum dielectric to approximate the effect of the solvent environment. Therefore, free energies can be computed without explicit thermodynamic sampling of the many possible configurations of the solvent molecules. The dielectric cavity, in which the solute resides, is determined self-consistently from the charge density of the solute.

$$
\varepsilon(n)=1+\left(\varepsilon_{b}-1\right) \frac{1}{2} \operatorname{erfc} \frac{\log \left(n / n_{c}\right)}{\sigma \sqrt{2}}
$$

where $n$ is the electron density, $\varepsilon_{\mathrm{b}}$ the bulk dielectric constant of the solvent, $n_{c}$ the critical density, and $\sigma$ the width of the transition which is chosen ${ }^{67}$ to be 0.6 to make the transition resolvable on typical real-space grids. The dielectric function turns on smoothly at a critical electron density value $n_{\mathrm{c}}$. This critical electron density, along with the effective surface tension of the solute-solvent interface $(\tau)$, is often fit to reproduce the solvation energies of molecules in that solvent. In this work, we do not perform this fit ourselves but rather use the values reported in earlier publications. ${ }^{67,68,70}\left(\right.$ For $\mathrm{H}_{2} \mathrm{O}$, we use $n_{\mathrm{c}}=$ $1.0 \times 10^{-3}$ and $\tau=9.5 \times 10^{-6}$, and for $\mathrm{CHCl}_{3}$, we use $n_{\mathrm{c}}=2.4 \times$ $10^{-5}$ and $\tau=-9.2 \times 10^{-6}$. All values are in atomic units.) At these values, the accuracy for neutral solutes have been observed $^{70}$ to be approximately $1 \mathrm{kcal} / \mathrm{mol}$. For a more detailed discussion of PCMs implemented in JDFTx, we refer the reader to refs 67,68 , and 70 .

We opted to use a nonlinear-response model over a linearresponse one because the electric fields near ionic crystals are often strong enough to access the regime where dielectric saturation effects become important for most solvents. ${ }^{67}$ In this regime, the rotational contribution to the dielectric function decreases with increasing external electric field. To capture this decrease in the dielectric response, the rotational contributions are separated from electronic and vibrational contributions and modeled as a field of interacting dipoles. Furthermore, nonlinear models avoid the numerical stability issues ${ }^{71}$ and the unphysically low (negative) solvation energies ${ }^{67}$ reported for the surfaces of ionic crystals.

Initial stoichiometric nanowire models with (001) and (101) facets were built from the anatase form of bulk $\mathrm{TiO}_{2}$. We refer to them as $\mathrm{nw}(001)$ and $\mathrm{nw}(101)$, respectively. Quasi-onedimensional anatase models with the dye adsorbates are placed in large periodic tetragonal super cells $\left(\sim 11 \times 22 \times 22 \AA^{3}\right)$. The periodic images of each nanowire are separated from each other to avoid any spurious interaction. Hence, large vacuum spaces of at least $20 \AA$ were introduced in the two translational directions perpendicular to the nanowire axes ([100] for $\mathrm{nw}(001)$ and [010] for $\mathrm{nw}(101))$. Similarly, periodicity is enlarged along the nanowire axis such that the dye adsorbate can be assumed to be isolated. The separation between the periodic replica of dye molecules along the nanowire axis is at least $8 \AA$. The Hellman-Feynmann forces on each atom have been minimized $(<0.01 \mathrm{eV} / \AA)$ based on the conjugategradients algorithm to fully optimize the initial geometries. A good convergence of the calculated properties with respect to the structural parameters, kinetic energy cutoff, and $k$-point sampling have been carefully checked. We repeated the same procedures and calculations separately with both the PBE and 
the HSE XC functional. The optimized nanowire models have maintained the anatase structure without any major reconstruction. The $\mathrm{Ti}-\mathrm{O}$ bonds on the facets get slightly larger while inner bonds are $\sim 1.95 \AA$ on average, reflecting the bulk value. The geometry optimization in both the PBE and the HSE cases relaxes surface atoms slightly out of their bulk positions without any major reconstruction. This prevents stress-related gap states from appearing in the band gap. Previously, we have also shown that further passivation of the facets is not required in the case of bare thin anatase nanowires. ${ }^{72}$ Therefore, the properties presented here are all referred to the fully optimized systems.

\section{RESULTS AND DISCUSSION}

The coumarin core $\left(\mathrm{C}_{9} \mathrm{H}_{6} \mathrm{O}_{2}\right)$ has been focused on as a candidate sensitizer for creating highly efficient DSSCs by many experimental and theoretical works. ${ }^{19,20,37,43-57}$ First of all, the adsorption of coumarin core on the $\mathrm{TiO}_{2}$ nanowires has been investigated as a minimal atomistic model to understand dye sensitization within the framework of total energy DFT calculations. We considered the coumarin molecule at various adsorption sites on the (001) and (101) facets of the anatase nanowires. The energetically favorable binding modes of coumarin for both of the cases are presented in Figure 1.

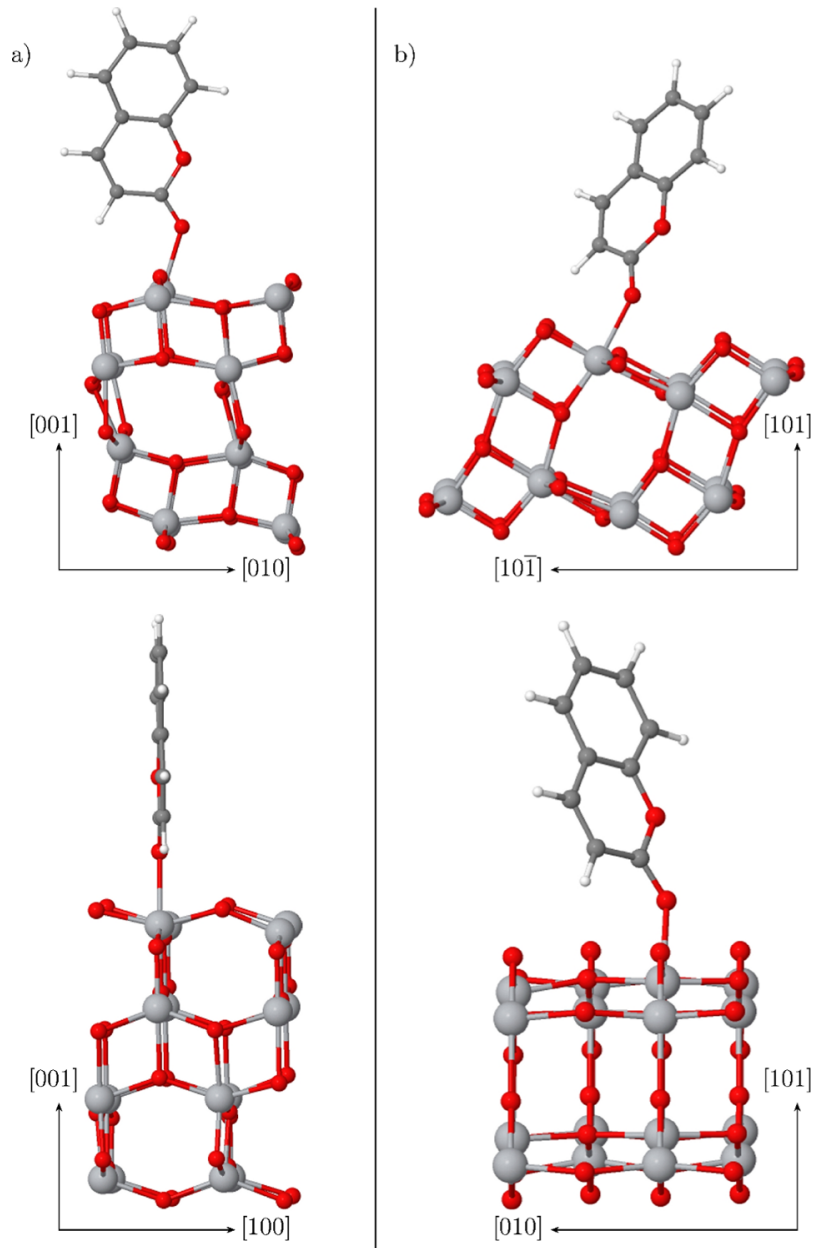

Figure 1. Optimized adsorption geometries of coumarin on anatase (001)-nanowire (left panel) and (101)-nanowire (right panel) shown from two different viewing orientations. Here, the red, light gray, dark gray, and white spheres represent $\mathrm{O}, \mathrm{Ti}, \mathrm{C}$, and $\mathrm{H}$ atoms, respectively.
Because of the tail oxygen of coumarin interacting with a 5-fold coordinated surface $\mathrm{Ti}$, the dye molecule aligns perpendicularly on both of the nanowire types forming a single bond (monodentate binding). Adsorption of coumarin does not cause noticeable distortion on the nanowire structure in both of the case. The length of the bond between the surface and the dye is 2.18 and $2.21 \AA$ in the cases of $\mathrm{nw}(101)$ and $\mathrm{nw}(001)$, respectively.

In the case of $\mathrm{C} 2-1$ dyes, the tail oxygen and the $\mathrm{OH}$ group play an important role in the adsorption on the nanowire surfaces. The optimized geometries of different binding modes of $\mathrm{C} 2-1$ on $\mathrm{TiO}_{2}$ nanowires are shown in Figure 2. It portrays two different adsorption modes. Similar to the coumarin case, one of them is the monodentate binding in which the tail oxygen forms a single bond with the surface $\mathrm{Ti}$ atom giving $\mathrm{C} 2$ 1 a perpendicular orientation with respect to the nanowire axis. In the second mode, in addition to the $\mathrm{O}-\mathrm{Ti}$ bond, the $\mathrm{OH}$ group loses the $\mathrm{H}$ to the nearest surface oxygen site, enabling the formation of a second $\mathrm{O}-\mathrm{Ti}$ bond between the dye and the nanowire. This bidentate mode is a chemical binding and so is much stronger than the monodentate case. For both of the nanowire types, the monodentate bond length is slightly larger than bidentate formation in which the bond lengths are $\sim 2.0 \AA$, which is a typical value of the bulk structures. The adsorption of C2-1 leads to only a minor change in the local environment on the nw(001). However, the surface oxygen on the nw(101) between the two $\mathrm{Ti}$ atoms forming the bidentate bonds buckles down slightly, as seen in Figure 2d.

We calculated the binding energies with a standard formulation as given in previous studies. ${ }^{28,29}$ These values are computed using PBE and HSE schemes and are presented in Table 1. The solvent effects with chloroform and water solutions have also been included on PBE results. Single bonding gives moderate and similar binding energies for both of the dyes on the two nanowire surface types. Regarding the binding energies, HSE functional yields values similar to the PBE values. Strong solution effects are found to drastically weaken the adsorption of the dye on the nanowire surface. This could indicate an expectation of low efficiencies for singly bound molecular cases because of the reduced stability. When the binding energies of the dyes on the nanowires in Table 1 are considered, one can say that the dissolution of the simple coumarin dye in the electrolyte is probable in applications, which causes a noticeable decrease in the photovoltaic performance of DSSCs. Therefore, conventional coumarin dyes (C343, NKX-2398, NKX-2510, etc.) are anchored to $\mathrm{TiO}_{2}$ through various carboxyl groups. In the tetrahydroquinoline case, the formation of the second bond as a result of the loss of the $\mathrm{H}$ in the $\mathrm{OH}$ group in the bidentate case of $\mathrm{C} 2-1$ dye enhances the binding appreciably. This bidentate mode of C2-1 gives the strongest binding energy among the cases considered here. Our PBE-calculated value of $0.94 \mathrm{eV}$ on $\mathrm{nw}(101)$ is in good agreement with generalized gradient approximation (GGA)-predicted binding energy of the isolated adsorption of the molecule on (101) slab surface by O'Rourke et al. ${ }^{42}$ The slight buckling of the surface oxygen on nw(101) brings an energetic penalty on the binding energy of C2-1 bidentate mode which leads to a reduction of the adsorption energy compared to that of the $\mathrm{nw}(001)$ case. Our results suggest that $\mathrm{C} 2-1$ is losing the $\mathrm{H}$ from its tail $\mathrm{OH}$ group to the nanowire surface to form a chemical bond. Therefore, it survives in a strongly ionic solution like water without dissociation. 
a)
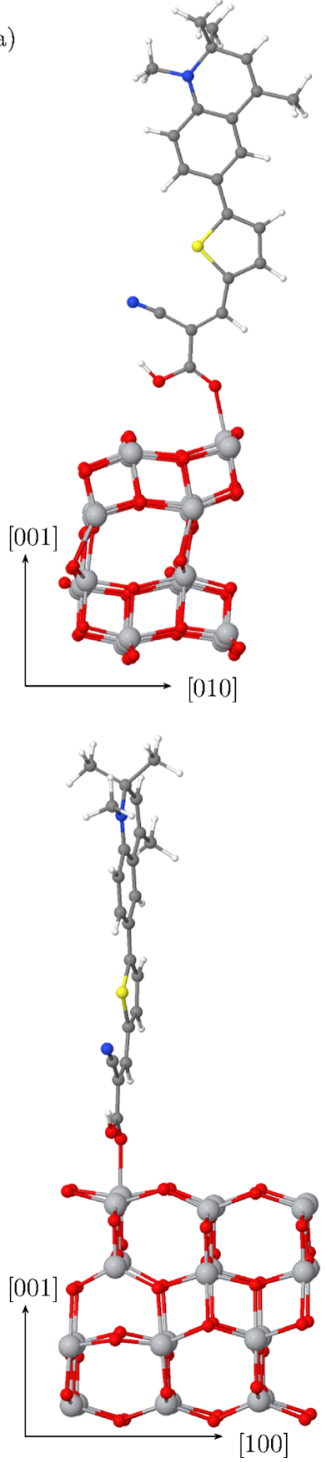

b)
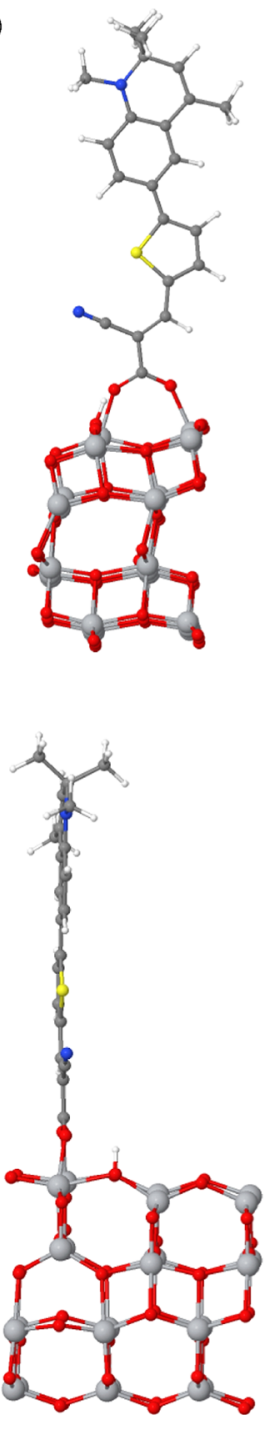

c)

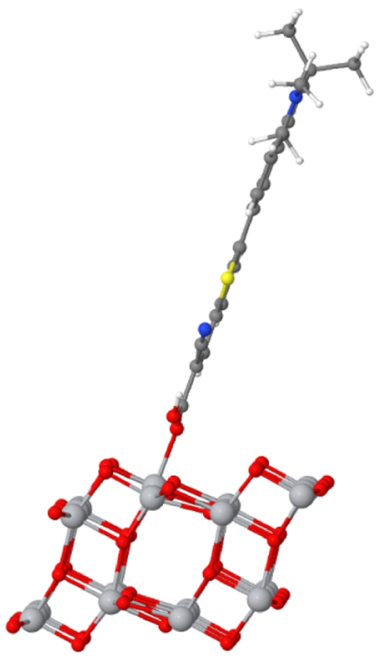

d)
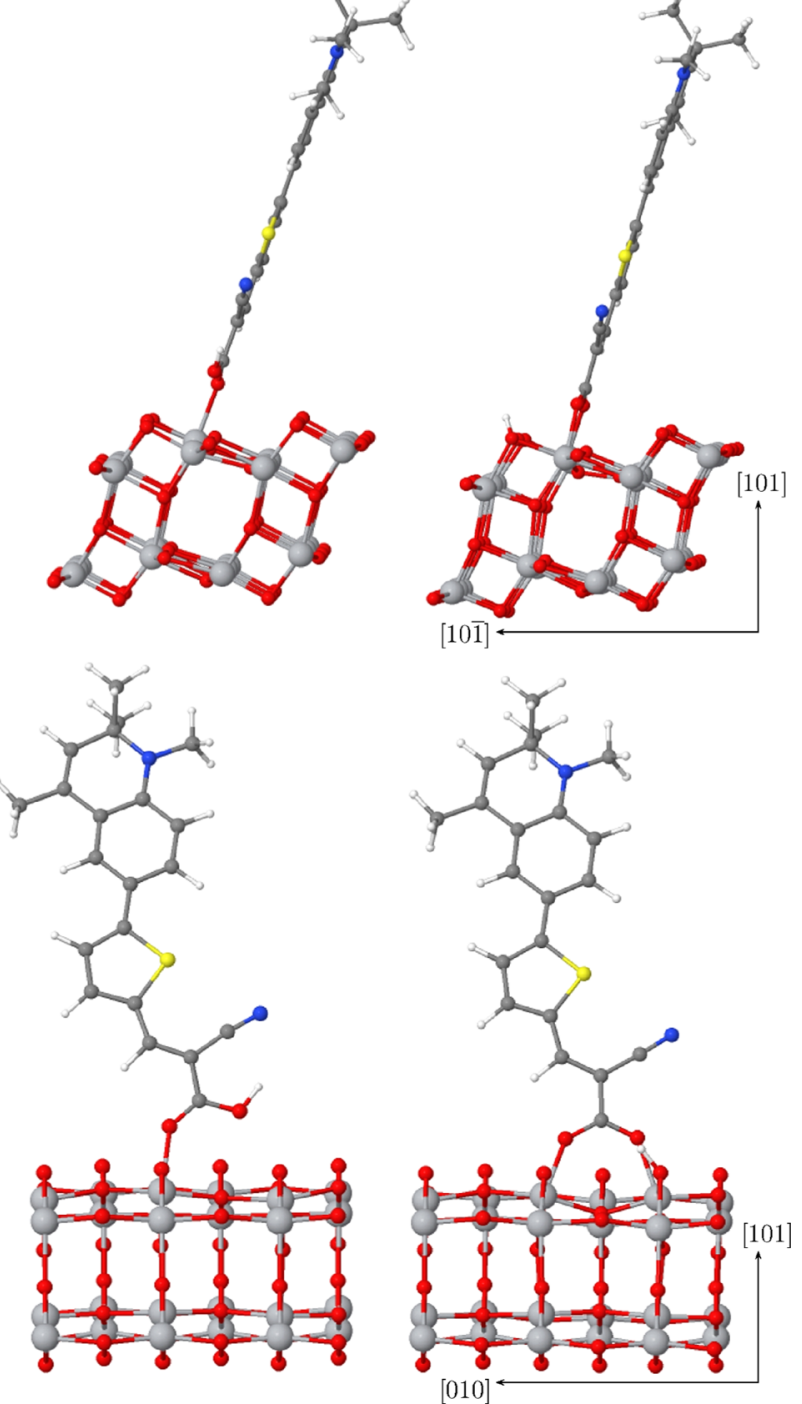

Figure 2. Optimized adsorption geometries of C2-1 on anatase (001)-nanowire (left panel) and (101)-nanowire (right panel) with two different viewing orientations for both monodentate and bidentate bonding modes. Here the red, light-gray, dark-gray, blue, yellow and white color spheres represent $\mathrm{O}, \mathrm{Ti}, \mathrm{C}, \mathrm{N}, \mathrm{S}$, and $\mathrm{H}$ atoms, respectively.

Table 1. Calculated Adsoprtion Energies of Dye-Nanowire Systems (eV)

\begin{tabular}{|c|c|c|c|c|c|c|c|c|}
\hline \multirow[b]{2}{*}{ dye } & \multicolumn{4}{|c|}{$@(001)$} & \multicolumn{4}{|c|}{$@(101)$} \\
\hline & PBE & HSE & $\mathrm{PBE}+\mathrm{PCM}^{a}$ & $\mathrm{PBE}+\mathrm{PCM}^{b}$ & PBE & HSE & $\mathrm{PBE}+\mathrm{PCM}^{a}$ & $\mathrm{PBE}+\mathrm{PCM}^{b}$ \\
\hline coumarin & -0.46 & -0.63 & -0.24 & -0.05 & -0.63 & -0.70 & -0.48 & -0.23 \\
\hline C2-1 (monodentate) & -0.72 & -0.73 & -0.71 & -0.22 & -0.57 & -0.62 & -0.44 & -0.10 \\
\hline C2-1 (bidentate) & -1.36 & -1.25 & -1.22 & -0.71 & -0.94 & -0.83 & -0.74 & -0.37 \\
\hline
\end{tabular}

When the solvent effect is included in the calculations, we notice that the binding energies change in the positive direction. This is because the binding sites on the $\mathrm{TiO}_{2}$ and the dye molecule interact very strongly with the solvent. This interaction is missing in vacuum calculations, resulting in a more negative binding energy. Because $\mathrm{H}_{2} \mathrm{O}$ is a more polar solvent than $\mathrm{CHCl}_{3}$ with a higher dielectric constant, it interacts more strongly with the binding sites; we therefore see that binding energies in $\mathrm{H}_{2} \mathrm{O}$ are more positive than the binding energies in $\mathrm{CHCl}_{3}$.
The density of states (DOS) of the dye-nanowire combined systems are presented in Figure 3. Our $\mathrm{TiO}_{2}$ nanowire models having diameters around $1 \mathrm{~nm}$ possess larger band gap values relative to the bulk and surface structures as a result of the quantum confinement effect. $^{72}$ In general, most of the deep lying occupied molecular orbitals stay in the valence band as a resonant state. Most importantly, a number of dye-related isolated and occupied states appear above the VB edge within the band gap, depending on the nature of the binding. As a result, the Fermi energy shifts up to higher energies, leading to an energy gap narrowing which is an important factor for 

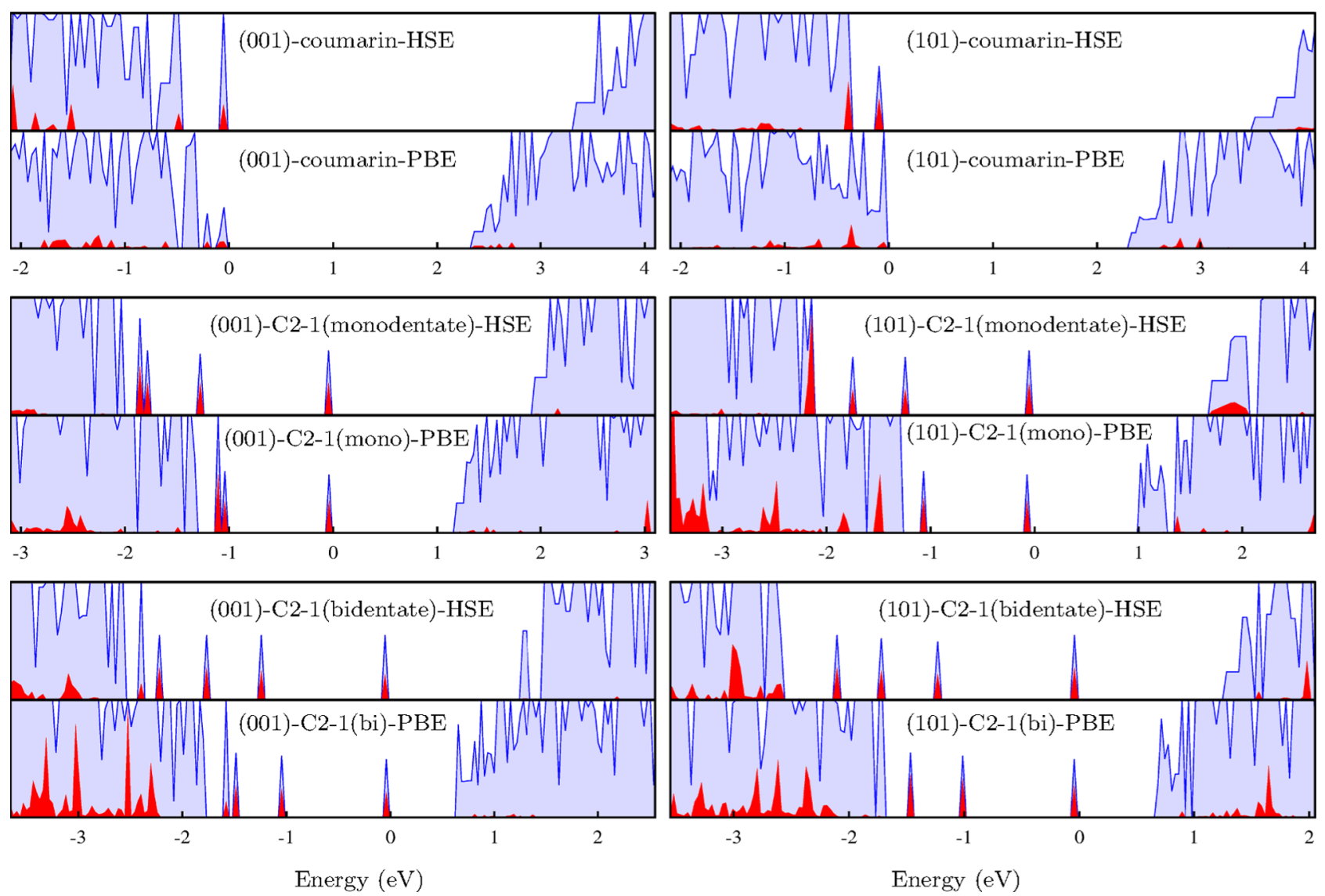

Figure 3. Density of states (DOS) of dye-nanowire combined systems. The contribution of the dye molecules are denoted as red shades by projecting on the dye molecular orbitals. The zero of the energy is set at the highest occupied energy level.

photovoltaic properties. Moreover, the lowest-lying unoccupied molecular levels of the dyes delocalize on the Ti $3 \mathrm{~d}$ states inside the conduction band of the nanowires. HSE functional heals the band gap underestimation which is inherent in the standard PBE XC scheme. The PBE-bandgaps of bare nw(101) and $\mathrm{nw}(001)$ are 2.51 and $2.69 \mathrm{eV}$, respectively. The HSE method gives an energy correction of $1.50 \mathrm{eV}$ for $\mathrm{nw}(101)$ and $1.37 \mathrm{eV}$ for the nw(001). This opening of the gap results in molecular states falling in the energy gap, which were previously in resonance with the VB at the PBE level. To compare PBE and HSE calculated DOS structures, we aligned them with respect to their deep core energy states. For both of the nanowires, the molecular states of coumarin appear around the VB edge while one of them is isolated from the rest in the case of HSE. For C2-1 monodentate mode, PBE predicts two isolated states above the VB. Because of the gap opening by the HSE functional, one more filled isolated state falls in the band gap of both of the nanowire types. On going from monodentate to bidentate bonding, there appears an additional isolated state above the VB. In the case of C2-1 bidentate mode, the positions and the number of dye-related energy levels calculated with the PBE functional are in agreement with the GGA results of O'Rourke et al. ${ }^{42}$ The only difference comes from the alignment of $\mathrm{TiO}_{2}$ energy bands with respect to the energy levels of the dye, which stems from our nanowire and their slab calculations. Significant band gap reduction is obtained in the case of C2-1 on both of the nanowires, which is important for light harvesting.

The absorption spectra of the sensitizers, coumarin, and C2-1 on $\mathrm{nw}(001)$ and $\mathrm{nw}(101)$ have been calculated at the PBE and
HSE levels using the formulation as described in our previous study on the bare anatase nanowires. ${ }^{72}$ The dye-related contributions in the optical spectra show characteristics similar to both PBE and HSE calculations, as shown in Figure 4. These features correspond to the first absorption peaks in each case associated with excitations from the highest occupied molecular states to the unoccupied molecular states which are coupled to the $\mathrm{CB}$ of the semiconductor. Similar absorption properties are obtained in the UV region, which are mostly related with the interband transitions from the VB to the $\mathrm{CB}$ states. Although the main characteristics are alike, PBE-calculated spectra are significantly red-shifted with respect to that of the HSE due to the local density approximation (LDA) giving rise to an inherent underestimation of the band gap of $\mathrm{TiO}_{2}$. The coumarin core brings the lowest-lying peak which extends the absorption threshold slightly into the visible region, as seen in Figure $4 \mathrm{a}$ for both nanowire types. The C2-1-nanowire combined system has optical properties that are more favorable than those of the coumarin-nanowire structures. A distinct and strong absorption peak results due to the excitation from the occupied molecular state at the Fermi energy to the frontier unoccupied molecular state which delocalizes inside the $\mathrm{CB}$ of the semiconductor. As a D- $\pi$-A type dye, such an excitation achieves charge redistribution from the donor to the acceptor moiety of C2-1 dye, which is important for the charge injection into the $\mathrm{CB}$ of $\mathrm{TiO}_{2}$. This peak associated with the chargetransfer (CT) state is identified at $\sim 2 \mathrm{eV}$ at the HSE level while PBE prediction falls in the near-infrared (IR) part of the spectrum. In the energetically most preferable bidentate bonding mode of C2-1 sensitizer, two absorption peaks are 
a)

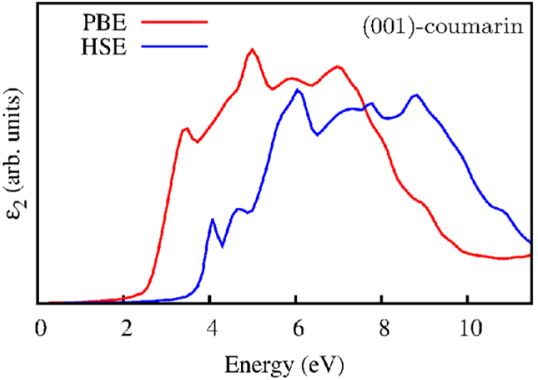

b)

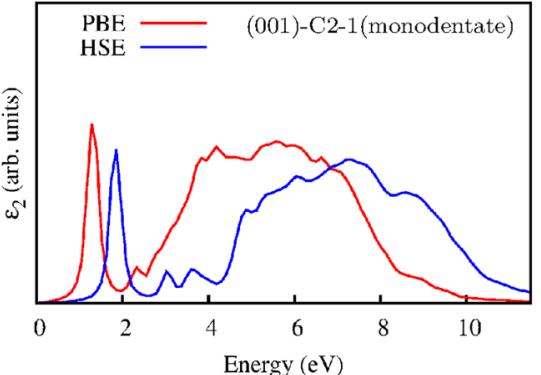

c)

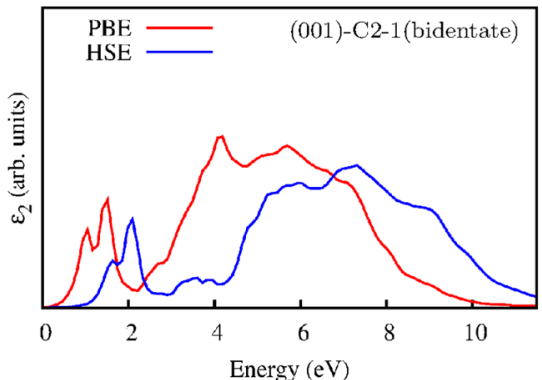

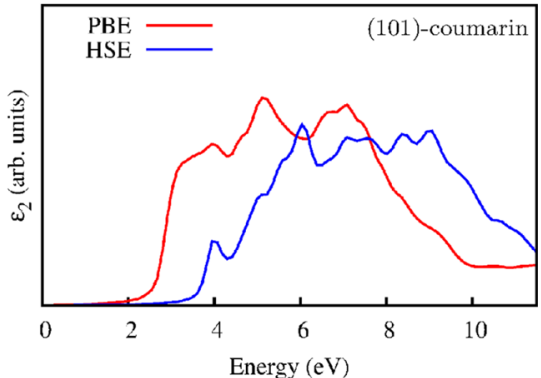
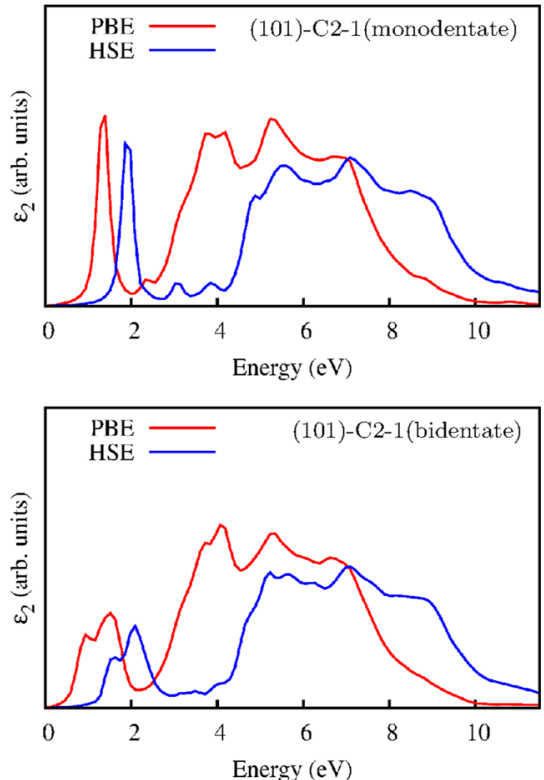

Figure 4. Absorption Spectra for (a) coumarin on anatase (001)-nanowire and (101)-nanowire (b) C2-1 monodentate adsorption on anatase (001)nanowire and (101)-nanowire (c) C2-1 bidentate bridging on anatase (001)-nanowire and (101)-nanowire, respectively.

identified which are significantly broadened in the visible range. This is reminiscent of the chemical nature of the adsorption of C2-1 on both the (001) and the (101) surfaces of the anatase nanowire. The first absorption peak positions of $\mathrm{C} 2-1$ on the anatase nanowire structures calculated at the HSE level are slightly red-shifted compared with the experimental data ${ }^{24,25}$ of Chen et al. obtained on nanocrystalline $\mathrm{TiO}_{2}$. Consequently, on the basis of our hybrid DFT computations, the bidentate bonding form of $\mathrm{C} 2-1$ on $\mathrm{TiO}_{2}$ survives in solution, causes a number of isolated filled molecular states to appear in the band gap, functionalizes the anatase nanowires to absorb a wide range of the visible region, and achieves charge separation, which is promising for both the enhancement of the charge injection efficiencies and for the reduction of the charge carrier recombination rates.

\section{CONCLUSIONS}

The binding geometries, electronic structures, and absorption characteristics of two organic molecules on $\mathrm{TiO}_{2}$ nanowires with (101) and (001) facets have been investigated using screened Coulomb hybrid density functional calculations. Coumarin causes new gap states to appear at the edge of the $\mathrm{VB}$ of the nanowires. These systems that we chose as the minimal dye-nanowire models result in a narrowing of the electronic band gap of $\mathrm{TiO}_{2}$. Strong bidentate binding of tetrahydroquinoline C2-1 dye brings a number of isolated and occupied gap states that both result in a significant narrowing of the band gap and cause a broader absorption structure functionalizing the semiconductor nanowires in the entire visible region. Nonlinear solvent effects suggest that the dissolution of coumarin and monodentate binding of C2-1 from the nanowires is probable in an actual electrolyte. Bidentate adsorption of donor- $\pi$-acceptor type $\mathrm{C} 2-1$ molecules can achieve directional charge-transfer excitation to increase charge injection probabilities, allow absorption in the full range of visible spectrum to achieve enhanced light harvesting, and exhibit strong binding to reduce degradation of possible device operation.

\section{AUTHOR INFORMATION}

\section{Corresponding Author}

*E-mail: emete@balikesir.edu.tr.

Notes

The authors declare no competing financial interest.

\section{ACKNOWLEDGMENTS}

This work is supported by TÜBİTAK, The Scientific and Technological Research Council of Turkey (Grant 110T394). Computational resources were provided by ULAKBİM, Turkish Academic Network \& Information Center.

\section{REFERENCES}

(1) O’Regan, B.; Grätzel, M. A Low-Cost, High-Efficiency Solar Cell Based on Dye-Sensitized Colloidal $\mathrm{TiO}_{2}$ Films. Nature (London, U.K.) 1991, 353, 737-740. 
(2) Fujishima, A.; Honda, K. Electrochemical Photolysis of Water at a Semiconductor Electrode. Nature (London, U.K.) 1972, 238, 37-38.

(3) Fujishima, A.; Zhang, X. T.; Tryk, D. A. TiO ${ }_{2}$ Photocatalysis and Related Surface Phenomena. Surf. Sci. Rep. 2008, 63, 515-582.

(4) Çakır, D.; Gülseren, O. First-Principles Study of Thin $\mathrm{TiO}_{x}$ and Bulklike Rutile Nanowires. Phys. Rev. B: Condens. Matter Mater. Phys. 2009, 80, 125424.

(5) Naicker, P. K.; Cummings, P. T.; Zhang, H.; Banfield, J. F. Characterization of Titanium Dioxide Nanoparticles Using Molecular Dynamics Simulations. J. Phys. Chem. B 2005, 109, 15243-15249.

(6) Iacomino, A.; Cantele, G.; Trani, F.; Ninno, D. DFT Study on Anatase $\mathrm{TiO}_{2}$ Nanowires: Structure and Electronic Properties As Functions of Size, Surface Termination, and Morphology. J. Phys. Chem. C 2010, 114, 12389-12400.

(7) Fuertes, V. C.; Negre, C. F. A.; Oviedo, M. B.; Bonafé, F. P.; Oliva, F. Y.; Sánchez, C. G. A Theoretical Study of the Optical Properties of Nanostructured $\mathrm{TiO}_{2}$. J. Phys.: Condens. Matter 2013, 25, 115304.

(8) Nazeeruddin, M. K.; Kay, A.; Rodicio, I.; Humphry-Baker, R.; Müller, E.; Liska, P.; Vlachopoulos, N.; Grätzel, M. Conversion of Light to Electricity by cis- $\mathrm{X}_{2} \operatorname{Bis}\left(2,2^{\prime}\right.$-bipyridyl-4,4'-dicarboxylate)ruthenium(II) Charge-Transfer Sensitizers (X $=\mathrm{Cl}-, \mathrm{Br}-, \mathrm{I}-, \mathrm{CN}-$, and SCN-) on Nanocrystalline Titanium Dioxide Electrodes. J. Am. Chem. Soc. 1993, 115, 6382-6390.

(9) Tachibana, Y.; Moser, J. E.; Grätzel, M.; Klug, D. R.; Durrant, J. R. Subpicosecond Interfacial Charge Separation in Dye-Sensitized Nanocrystalline Titanium Dioxide Films. J. Phys. Chem. 1996, 100, 20056-20062.

(10) Thompson, D. W.; Wishart, J. F.; Brunschwig, B. S.; Sutin, N. Efficient Generation of the Ligand Field Excited State of Tris- $\left(2,2^{\prime}-\right.$ bipyridine)-ruthenium(II) through Sequential Two-Photon Capture by $[\mathrm{Ru}(\text { bpy }) 3]^{2+}$ or Electron Capture by $[\mathrm{Ru}(\text { bpy }) 3]^{3+}$. J. Phys. Chem. A 2001, 105, 8117-8122.

(11) Nakade, S.; Kubo, W.; Saito, Y.; Kamzaki, T.; Kitamura, T.; Wada, Y.; Yanagida, S. J. Influence of Measurement Conditions on Electron Diffusion in Nanoporous $\mathrm{TiO}_{2}$ Films: Effects of Bias Light and Dye Adsorption. J. Phys. Chem. B 2003, 107, 14244-14248.

(12) Wang, P.; Zakeeruddin, S. M.; Moser, J. E.; Nazeeruddin, M. K.; Sekiguchi, T.; Grätzel, M. A Stable Quasi-Solid-State Dye-Sensitized Solar Cell with an Amphiphilic Ruthenium Sensitizer and Polymer Gel Electrolyte. Nat. Mater. 2003, 2, 402-407.

(13) Benkö, G.; Kallioinen, J.; Myllyperkiö, P.; Trif, F.; KorppiTommola, J. E. I.; Yartsev, A. P.; Sundström, V. Interligand Electron Transfer Determines Triplet Excited State Electron Injection in $\mathrm{RuN}_{3}$ Sensitized $\mathrm{TiO}_{2}$ Films. J. Phys. Chem. B 2004, 108, 2862-2867.

(14) Wang, P.; Klein, C.; Humphry-Baker, R.; Zakeeruddin, S.; Grätzel, M. Stable $\geq 8 \%$ Efficient Nanocrystalline Dye-Sensitized Solar Cell Based on an Electrolyte of Low Volatility. Appl. Phys. Lett. 2005, 86,123508 .

(15) Nazeeruddin, M. K.; De Angelis, F.; Fantacci, S.; Selloni, A.; Viscardi, G.; Liska, P.; Ito, S.; Takeru, B.; Grätzel, M. Combined Experimental and DFT-TDDFT Computational Study of Photoelectrochemical Cell Ruthenium Sensitizers. J. Am. Chem. Soc. 2005, 127, 16835-16847.

(16) Mishra, A.; Fischer, M. K. R.; Bäuerle, P. Metal-Free Organic Dyes for Dye-Sensitized Solar Cells: From Structure: Property Relationships to Design Rules. Angew. Chem., Int. Ed. 2009, 48, 2474-2499.

(17) Khazraji, A. C.; Hotchandani, S.; Das, S.; Kamat, P. V. Controlling Dye (Merocyanine-540) Aggregation on Nanostructured $\mathrm{TiO}_{2}$ Films. An Organized Assembly Approach for Enhancing the Efficiency of Photosensitization. J. Phys. Chem. B 1999, 103, 46934700.

(18) Sayama, K.; Hara, K.; Mori, N.; Satsuki, M.; Suga, S.; Tsukagoshi, S.; Abe, Y.; Sugihara, H.; Arakawa, H. Photosensitization of a Porous $\mathrm{TiO}_{2}$ Electrode with Merocyanine Dyes Containing a Carboxyl Group and a Long Alkyl Chain. Chem. Commun. (Cambridge, U.K.) 2000, 1173-1174.
(19) Hara, K.; Wang, Z.-S.; Sato, T.; Furube, A.; Katoh, R.; Sugihara, H.; Dan-oh, Y.; Kasada, C.; Shinpo, A.; Suga, S. OligothiopheneContaining Coumarin Dyes for Efficient Dye-Sensitized Solar Cells. J. Phys. Chem. B 2005, 109, 15476-15482.

(20) Wang, Z.-S.; Cui, Y.; Dan-oh, Y.; Kasada, C.; Shinpo, A.; Hara, K. Molecular Design of Coumarin Dyes for Stable and Efficient Organic Dye-Sensitized Solar Cells. J. Phys. Chem. C 2008, 112, 17011-17017.

(21) Qin, H.; Wenger, S.; Xu, M.; Gao, F.; Jing, X.; Wang, P.; Zakeeruddin, S. M.; Grätzel, M. An Organic Sensitizer with a Fused Dithienothiophene Unit for Efficient and Stable Dye-Sensitized Solar Cells. J. Am. Chem. Soc. 2008, 130, 9202-9203.

(22) Koumura, N.; Wang, Z.; Mori, S.; Miyashita, M.; Suzuki, E.; Hara, K. Alkyl-Functionalized Organic Dyes for Efficient Molecular Photovoltaics. J. Am. Chem. Soc. 2006, 128, 14256-14257. Correction: 2008, 130, 4202-4203.

(23) Jiang, X.; Marinado, T.; Gabrielsson, E.; Hagberg, D. P.; Sun, L.; Hagfeldt, A. Structural Modification of Organic Dyes for Efficient Coadsorbent-Free Dye-Sensitized Solar Cells. J. Phys. Chem. C 2010, $114,2799-2805$.

(24) Chen, R.; Yang, X.; Tian, H.; Wang, X.; Hagfeldt, A.; Sun, L. Effect of Tetrahydroquinoline Dyes Structure on the Performance of Organic Dye-Sensitized Solar Cells. Chem. Mater. 2007, 19, 40074015.

(25) Chen, R.; Yang, X.; Tian, H.; Sun, L. Tetrahydroquinoline Dyes with Different Spacers for Organic Dye-Sensitized Solar Cells. J. Photochem. Photobiol., A 2007, 189, 295-300.

(26) Ito, S.; Miura, H.; Uchida, S.; Takata, M.; Sumioka, K.; Liska, P.; Comte, P.; Pëchy, P.; Grätzel, M. High-Conversion-Efficiency Organic Dye-Sensitized Solar Cells with a Novel Indoline Dye. Chem. Commun. (Cambridge, U.K.) 2008, 5194-5196.

(27) Mete, E.; Uner, D.; Cakmak, M.; Gülseren, O.; Ellialtoğlu, Ş. Effect of Molecular and Electronic Structure on the Light-Harvesting Properties of Dye Sensitizers. J. Phys. Chem. C 2007, 111, 7539-7547.

(28) Çakır, D.; Gülseren, O.; Mete, E.; Ellialtoğlu, Ş Dye Adsorbates BrPDI, BrGly, and BrAsp on Anatase $\mathrm{TiO}_{2}(001)$ for Dye-Sensitized Solar Cell Applications. Phys. Rev. B: Condens. Matter Mater. Phys. 2009, 80, 035431.

(29) Çakır, D.; Gülseren, O.; Mete, E.; Ellialtoğlu, Ş Interaction of BrPDI, BrGly, and BrAsp with the Rutile $\mathrm{TiO}_{2}(110)$ Surface for Photovoltaic and Photocatalytic Applications: A First-Principles Study. J. Phys. Chem. C 2011, 115, 9220-9226.

(30) Daul, C.; Baerends, E. J.; Vernooijs, P. A Density Functional Study of the MLCT States of $\left[\mathrm{Ru}(\mathrm{bpy})_{3}\right]^{2+}$ in $D_{3}$ Symmetry. Inorg. Chem. 1994, 33, 3538-3543.

(31) Brocks, G.; Tol, A. Small Band Gap Semiconducting Polymers Made from Dye Molecules: Polysquaraines. J. Phys. Chem. 1996, 100, $1838-1846$.

(32) Naito, K.; Sakurai, M.; Egusa, S. Molecular Design, Syntheses, and Physical Properties of Nonpolymeric Amorphous Dyes for Electron Transport. J. Phys. Chem. A 1997, 101, 2350-2357.

(33) Gorelsky, S. I.; Lever, A. B. P. Electronic Structure and Spectra of Ruthenium Diimine Complexes by Density Functional Theory and INDO/S. Comparison of the Two Methods. J. Organomet. Chem. 2001, 635, 187-196.

(34) Stier, W.; Prezhdo, O. V. Nonadiabatic Molecular Dynamics Simulation of Light-Induced Electron Transfer from an Anchored Molecular Electron Donor to a Semiconductor Acceptor. J. Phys. Chem. B 2002, 106, 8047-8054.

(35) Acebal, P.; Blaya, S.; Carretero, L. Ab Initio Study of Absorption and Emission Spectra of PM567. Chem. Phys. Lett. 2003, 374, 206214.

(36) Pasveer, W. F.; Bobbert, P. A.; Michels, M. A. J.; LangeveldVoss, B. M. W.; Schoo, H. F. M.; Bastiaansen, J. J. A. M. Ab Initio Study of Energy-Level Alignments in Polymer-Dye Blends. Chem. Phys. Lett. 2003, 381, 392-396.

(37) Hara, K.; Sato, T.; Katoh, R.; Furube, A.; Ohga, Y.; Shinpo, A.; Suga, S.; Sayama, K.; Sugihara, H.; Arakawa, H. Molecular Design of 
Coumarin Dyes for Efficient Dye-Sensitized Solar Cells. J. Phys. Chem. B 2003, 107, 597-606.

(38) Bañuelos Prieto, J.; López Arbeloa, F.; Martínez Martínez, V.; López Arbeloa, I. Theoretical Study of the Ground and Excited Electronic States of Pyrromethene 546 Laser Dye and Related Compounds. Chem. Phys. 2004, 296, 13-22.

(39) Campbell, L.; Mukamel, S. Simulation of X-Ray Absorption Near Edge Spectra of Electronically Excited Ruthenium Tris-2,2'bipyridine. J. Chem. Phys. 2004, 121, 12323.

(40) Persson, P.; Lundqvist, M. J.; Ernstorfer, R.; Goddard, W. A., III; Willig, F. Quantum Chemical Calculations of the Influence of AnchorCum-Spacer Groups on Femtosecond Electron Transfer Times in Dye-Sensitized Semiconductor Nanocrystals. J. Chem. Theory Comput. 2006, 2, 441-451.

(41) Zhang, C.-R.; Liu, L.; Zhe, J.-W.; Jin, N.-Z.; Ma, Y.; Yuan, L.-H.; Zhang, M.-L.; Wu, Y.-Z.; Liu, Z.-J.; Chen, H.-S. The Role of the Conjugate Bridge in Electronic Structures and Related Properties of Tetrahydroquinoline for Dye Sensitized Solar Cells. Int. J. Mol. Sci. 2013, 14, 5461-5481.

(42) O'Rourke, C.; Bowler, D. R. Adsorption of ThiopheneConjugated Sensitizers on $\mathrm{TiO}_{2}$ Anatase (101). J. Phys. Chem. C 2010, 114, 20240-20248.

(43) Kitamura, T.; Ikeda, M.; Shigaki, K.; Inoue, T.; Anderson, N. A.; Ai, X.; Lian, T.; Yangagida, S. Phenyl-Conjugated Oligoene Sensitizers for $\mathrm{TiO}_{2}$ Solar Cells. Chem. Mater. 2004, 16, 1806-1812.

(44) Hara, K.; Miyamoto, K.; Abe, Y.; Yanagida, M. Electron Transport in Coumarin-Dye-Sensitized Nanocrystalline $\mathrm{TiO}_{2}$ Electrodes. J. Phys. Chem. B 2005, 109, 23776-23778.

(45) Li, S.-L.; Jiang, K.-J.; Shao, K.-F.; Yang, L.-M. Novel Organic Dyes for Efficient Dye-Sensitized Solar Cells. Chem. Commun. (Cambridge, U.K.) 2006, 2792-2794.

(46) Chiba, Y.; Islam, A.; Watanabe, Y.; Komiya, R.; Koide, N.; Han, L. Dye-Sensitized Solar Cells with Conversion Efficiency of 11.1\%. Jpn. J. Appl. Phys. 2006, 45, L638-L640.

(47) Wang, Z.-S.; Cui, Y.; Hara, K.; Dan-oh, Y.; Kasada, C.; Shinpo, A. A High-Light-Harvesting-Efficiency Coumarin Dye for Stable DyeSensitized Solar Cells. Adv. Mater. (Weinheim, Ger.) 2007, 19, 11381141.

(48) Wang, Z.-S.; Cui, Y.; Dan-oh, Y.; Kasada, C.; Shinpo, A.; Hara, K. Thiophene-Functionalized Coumarin Dye for Efficient DyeSensitized Solar Cells: Electron Lifetime Improved by Coadsorption of Deoxycholic Acid. J. Phys. Chem. C 2007, 111, 7224-7230.

(49) Kurashige, Y.; Nakajima, T.; Kurashige, S.; Hirao, K. Theoretical Investigation of the Excited States of Coumarin Dyes for DyeSensitized Solar Cells. J. Phys. Chem. A 2007, 111, 5544-5548.

(50) Preat, J.; Loos, P.-F.; Assfeld, X.; Jacquemin, D.; Perpète, E. A. A TD-DFT Investigation of UV Spectra of Pyranoidic Dyes: A NCM vs PCM Comparison. THEOCHEM 2007, 808, 85-91.

(51) Wong, B. M.; Cordaro, J. G. Coumarin Dyes for Dye-Sensitized Solar Cells: A Long-Range-Corrected Density Functional Study. J. Chem. Phys. 2008, 129, 214703.

(52) Zhang, X.; Zhang, J.-J.; Xia, Y.-Y. Molecular Design of Coumarin Dyes with High Efficiency in Dye-Sensitized Solar Cells. J. Photochem. Photobiol., A 2008, 194, 167-172.

(53) Sánchez-de-Armas, R.; San Miguel, M. A.; Oviedo, J.; Sanz, J. F. Coumarin Derivatives for Dye Sensitized Solar Cells: A TD-DFT Study. Phys. Chem. Chem. Phys. 2012, 14, 225-233.

(54) Sánchez-de-Armas, R.; San Miguel, M. A.; Oviedo, J.; Sanz, J. F. Molecular Modification of Coumarin Dyes for More Efficient Dye Sensitized Solar Cells. J. Chem. Phys. 2012, 136, 194702.

(55) Oprea, C. I.; Panait, P.; Cimpoesu, F.; Ferbinteanu, M.; Girtu, M. A. Density Functional Theory (DFT) Study of Coumarin-Based Dyes Adsorbed on $\mathrm{TiO}_{2}$ Nanoclusters-Applications to DyeSensitized Solar Cells. Materials 2003, 6, 2372-2392.

(56) Faber, C.; Duchemin, I.; Deutsch, T.; Blase, X. Many-Body Green's Function Study of Coumarins for Dye-Sensitized Solar Cells. Phys. Rev. B: Condens. Matter Mater. Phys. 2012, 86, 155315.
(57) Verma, S.; Ghosh, H. N. Tuning Interfacial Charge Separation by Molecular Twist: A New Insight into Coumarin-Sensitized $\mathrm{TiO}_{2}$ Films. J. Phys. Chem. C 2014, 118, 10661-10669.

(58) Perdew, J. P.; Burke, K.; Ernzerhof, M. Generalized Gradient Approximation Made Simple. Phys. Rev. Lett. 1996, 77, 3865-3868.

(59) Heyd, J.; Scuseria, G. E.; Ernzerhof, M. Hybrid Functionals Based on a Screened Coulomb Potential. J. Chem. Phys. 2003, 118, 8207.

(60) Heyd, J.; Scuseria, G. E.; Ernzerhof, M. Erratum: Hybrid Functionals Based on a Screened Coulomb Potential J. Chem. Phys. 2006, 124, 219906.

(61) Paier, J.; Marsman, M.; Hummer, K.; Kresse, G.; Gerber, I. C.; Ángyán, J. G. Erratum: Screened Hybrid Density Functionals Applied to Solids. J. Chem. Phys. 2006, 125, 249901.

(62) Kresse, G.; Hafner, J. Ab Initio Molecular Dynamics for Liquid Metals. Phys. Rev. B: Condens. Matter Mater. Phys. 1993, 47, 558-561.

(63) Blöchl, P. E. Projector Augmented-Wave Method. Phys. Rev. B: Condens. Matter Mater. Phys. 1994, 50, 17953-17978.

(64) Kresse, G.; Joubert, J. From Ultrasoft Pseudopotentials to the Projector Augmented-Wave Method. Phys. Rev. B: Condens. Matter Mater. Phys. 1999, 59, 1758-1775.

(65) Perdew, J. P.; Ernzerhof, M.; Burke, K. Rationale for Mixing Exact Exchange with Density Functional Approximations. J. Chem. Phys. 1996, 105, 9982-9985.

(66) Letchworth-Weaver, K.; Arias, T. A. Joint Density Functional Theory of the Electrode-Electrolyte Interface: Application to Fixed Electrode Potentials, Interfacial Capacitances, and Potentials of Zero Charge. Phys. Rev. B: Condens. Matter Mater. Phys. 2012, 86, 075140.

(67) Gunceler, D.; Letchworth-Weaver, K.; Sundararaman, R.; Schwarz, K. A.; Arias, T. A. The Importance of Nonlinear Fluid Response in Joint Density-Functional Theory Studies of Battery Systems. Modell. Simul. Mater. Sci. Eng. 2013, 21, 074005.

(68) Gunceler, D.; Arias., T. A. Universal Iso-Density Polarizable Continuum Model for Molecular Solvents. 2014, arXiv:1403.6465.

(69) Sundararaman, R.; Gunceler, D.; Letchworth-Weaver, K.; Arias, T. A. JDFTx. http://jdftx.sourceforge.net, 2012.

(70) Sundararaman, R.; Gunceler, D.; Arias, T. A. Weighted-Density Functionals for Cavity Formation and Dispersion Energies in Continuum Solvation Models. 2014, arXiv:1407.4011.

(71) Sánchez, V. M.; Sued, M.; Scherlis, D. A. First-Principles Molecular Dynamics Simulations at Solid-Liquid Interfaces with a Continuum Solvent. J. Chem. Phys. 2009, 131, 174108.

(72) Ünal, H.; Gülseren, O.; Ellialtoğlu, Ş.; Mete, E. Electronic Structures and Optical Spectra of Thin Anatase $\mathrm{TiO}_{2}$ Nanowires through Hybrid Density Functional and Quasiparticle Calculations. Phys. Rev. B: Condens. Matter Mater. Phys. 2014, 89, 205127. 